\title{
Spectral Sensitivity of Circadian, Neuroendocrine and Neurobehavioral Effects of Light
}

\author{
Steven W. LOCKLEY \\ Division of Sleep Medicine, Brigham and Women's Hospital, and \\ Division of Sleep Medicine, Harvard Medical School, Boston, USA, \\ 221 Longwood Avenue, Boston, MA 02115, USA \\ E-mail: slockley@hms.harvard.edu
}

(received on November 5, 2007, accepted on November 30, 2007)

\begin{abstract}
The mammalian eye detects light for a range of behavioral and physiological responses separate and apart from sight. In humans, ocular light exposure resets the endogenous circadian pacemaker, suppresses synthesis of the pineal hormone melatonin, enhances morning cortisol production, increases heart rate and core body temperature, induces pupillary constriction, and improves subjective and objective measures of alertness. Several lines of evidence suggest that these responses are mediated primarily via a novel photoreception system with short-wavelength sensitivity $\left(\lambda_{\max } 460-480 \mathrm{~nm}\right)$ different from that used for sight. In order to compare the relative contribution of the novel photoreceptor system with the three-cone visual photopic system for multiple non-visual effects of light, we compared the responses following exposure to an equal photon density $\left(2.8 \times 10^{13}\right.$ photons $\left./ \mathrm{cm}^{2} / \mathrm{s}\right)$ either $460 \mathrm{~nm}(n=8)$ or $555 \mathrm{~nm}(n=8)$ monochromatic light $(10 \mathrm{~nm}$ half-peak bandwidth) at night on circadian phase resetting, melatonin suppression and subjective and objective correlates of alertness. As compared to $555 \mathrm{~nm}$ exposure, $460 \mathrm{~nm}$ light caused twice the phase shift in the circadian melatonin rhythm, double the amount of melatonin suppression, and significantly reduced auditory reaction time and lapses of attention. Exposure to $460 \mathrm{~nm}$ light also preferentially suppressed delta-theta $(0.5-5.5 \mathrm{~Hz})$ activity and elevated high alpha power $(9.5-10.5 \mathrm{~Hz})$ in the waking electroencephalogram (EEG) recordings, indicating a more alert state. The short-wavelength sensitivity to the non-visual effects of light indicates that the photopic visual system is not the primary photoreceptor system mediating these responses to light, and is consistent with predominant input from the novel melanopsin-containing photosensitive retinal ganglion cells. These data also suggest that short-wavelength light may be an effective therapy for resetting the circadian system in Circadian Rhythm Sleep Disorders and could be used as a direct fatigue countermeasure in a range of clinical and occupational settings. Translation of these basic findings into real-world applications is beginning to occur but the challenge to architects and lighting designers is to provide lighting that optimizes both the visual and non-visual effects of light simultaneously and safely.
\end{abstract}

Key words: light, circadian, melatonin, alertness, performance

\section{Introduction}

Much like the ear has dual functions for audition and balance, the human eye has a dual role in detecting light for a range of behavioral and physiological responses separate and apart from sight. Ocular light exposure induces multiple neuroendocrine, neurobehavioral and physiological responses including melatonin suppression, pupillary constriction, heart rate elevation, enhancement of alertness and performance, changes in brain activity patterns, phase-shifts of the endogenous circadian pacemaker and even stimula- tion of circadian clock gene expression (Campbell et al., 1995; Czeisler \& Wright, 1999; Cajochen et al., 2000; Lockley et al., 2003; Phipps-Nelson et al., 2003; Cajochen et al., 2005; Cajochen et al., 2006; Lockley et al., 2006). For want of a more appropriate expression, these wide-ranging effects of light are collectively called 'non-visual' or 'non-image forming' responses and are sometimes grouped under the term 'circadian photoreception', as much of the behavioral and neuroanatomical work that first identified these effects was focused on studies of the ability of light to shift 
the timing of the circadian pacemaker. While our basic understanding of these responses has been derived largely from tracing and gene ablation experiments in rodents (see Peirson \& Foster, 2006 for review), parallel studies in humans have also characterized some of the properties of this novel photoreceptor system and are leading to the first attempts to translate these basic findings into real-world applications.

The impact of different types of blindness on the non-visual effects of light has been used as an indirect method to assess the relative contribution of the visual photoreceptors systems in mediating these responses. Firstly, these studies have shown that eyes are required: Individuals without eyes, either through bilateral enucleation or as a result of developmental disorders, are unable to entrain their circadian pacemaker to the 24-hour light-dark cycle or have their melatonin synthesis suppressed by light (Czeisler et al., 1995; Lockley et al., 1997). Secondly, disorders of the visual system do not always attenuate the non-visual effects of light. The majority of visually impaired individuals who maintain some degree of light perception, even with little usable vision in some cases, have normally-phased circadian rhythms (Lockley et al., 1997). Primary loss of the central or peripheral visual fields is also not associated with circadian rhythm disorders, suggesting that the nonvisual photoreceptor system is less sensitive to the spatial distribution and intensity of light. Color blindness, a more specific lesion of the photopic visual system, also does not attenuate non-visual responses to light, as assessed by melatonin suppression ( $\mathrm{Ru}-$ berg et al., 1996). Finally, and most definitively, it has been demonstrated that some totally visually blind people maintain circadian phase-shifting and melatonin suppression responses to light (Czeisler et al., 1995; Klerman et al., 2002; Hull et al., 2004) even in the absence of any measurable visual response, as assessed by visually-evoked potentials or electroretinogram (Czeisler et al., 1995). Thus, non-visual effects of light can occur in humans without a functional visual system, as has also been shown in rodents (Hattar et al., 2003).

Determining the spectral, or wavelength, sensitivity of non-visual responses to light is another method by which to characterize the photoreceptor(s) mediating such responses in humans. Two action spectra for the melatonin suppressing effects of light indicate a peak sensitivity in the short-wavelength (blue) range ( $\sim 460 \mathrm{~nm}$ ) (Brainard et al., 2001; Thapan et al., 2001), that does not match the action spectra for human scotopic or photopic vision, or the absorption spectra for the individual red, green or blue cones. These findings are consistent, however, with the action spectra for a range of non-visual effects of light in rodents (see Brainard \& Hanifin, 2005 for review) and with the spectral sensitivity of intrinsically-photosensitive melanopsin-containing ganglion cells that mediate non-visual effects of light in rodents and primates $\left(\lambda_{\max } \sim 480 \mathrm{~nm}\right)$ (Gooley et al., 2001; Berson et al., 2002; Dacey et al., 2005). Melanopsin is also present in human eyes (Provencio et al., 2000).

The aim of the current experiment was to quantify the relative contribution of the novel non-rod, noncone melanopsin-mediated photoreceptor system and the three-cone photopic visual system to a range of circadian, neuroendocrine and neurobehavioral responses in humans. We therefore chose to compare the effects of exposure to an equal photon density of $460 \mathrm{~nm}$ light, close to the peak sensitivity of the melanopsin-mediated photoreceptor system, and $555 \mathrm{~nm}$ light, the peak sensitivity of the three-cone photopic system.

\section{Methods}

We studied 16 healthy subjects (8F; mean age \pm $\mathrm{SD}=23.3 \pm 2.4 \mathrm{yrs}$; range $19-27 \mathrm{yrs})$ in the Intensive Physiology Monitoring (IPM) Unit at the Brigham and Women's Hospital. The study was approved by an Institutional Review Board and subjects gave written informed consent. The pre-study screening and inpatient study protocol and conditions are detailed elsewhere (Zeitzer et al., 2000; Lockley et al., 2003; 2006). Briefly, subjects were studied for nine days in an environment free of time cues. Following a 3-day baseline (16:8h wake: sleep), subjects underwent a $\sim 50$-hour Constant Routine procedure (CR) and 8-h recovery sleep before the 16-h light-exposure 'day'. Subjects were exposed to monochromatic light for 6.5 hours, centered in the 16-h waking episode. Subjects then slept for 8 hours prior to starting a second $\sim 30$ h CR, followed by a further 8-hour sleep opportunity before discharge. Ambient room lighting during scheduled wake episodes on baseline days 1-3 was $\sim 190$ lux maximum until midway through the third baseline day, when ambient lighting was maintained at $<2$ lux until the end of the study. Monochromatic light exposure $(6.5 \mathrm{~h})$ was timed to start $9.25 \mathrm{~h}$ before respective waketime during each subjects' baseline days, corresponding on average to $\sim 6.75 \mathrm{~h}$ before core body temperature minimum, a phase at which white light exposure induces robust phase delays (Zeitzer et al., 2000). Monochromatic light was generated using a 1,200 W xenon arc lamp and grating monochromator and administered via a modified Ganzfeld source coated with 96-99\% reflective paint, described in detail elsewhere (Brainard et al., 2001). Fifteen minutes prior to expo- 
sure, a pupil dilator was administered to each eye after which time subjects wore black-out goggles until the start of the light exposure. Subjects were randomized for exposure to either $460 \mathrm{~nm}(n=8)$ or $555 \mathrm{~nm}(n=8)$ monochromatic light $( \pm 10 \mathrm{~nm}$ halfpeak bandwidth) of equal photon density $\left(2.8 \times 10^{13}\right.$ photons $/ \mathrm{cm}^{2} / \mathrm{s}$ ), corresponding to an irradiance of $12.1 \mu \mathrm{W} / \mathrm{cm}^{2}$ and $10.0 \mu \mathrm{W} / \mathrm{cm}^{2}$ and an illuminance of 5 lux and 68 photopic lux, respectively (for additional details, see Lockley et al., 2003; 2006).

Study measures included plasma melatonin sampled every 20-60 minutes throughout the study, continuous polysomnographic recordings from the $z$-line (Fz-Ax, Cz-Ax, Pz-Ax, and Oz-Ax) throughout the $\mathrm{CR}$ and light-exposure episodes and a neurobehavioral performance battery every 30-120 minutes when awake including the Karolinksa Sleepiness Scale to assess subjective alertness, a 10-minute auditory psychomotor vigilance test and a Karolinska Drowsiness Test (KDT). Circadian phase assessments were made by monitoring the melatonin secretory profile during two constant routine (CR) procedures (Duffy and Dijk, 2005), before and after exposure to monochromatic light.

Phase shifts (mean $\pm \mathrm{SD}$ ) were calculated as the difference in clock time between initial and final phase of the melatonin rhythm measured during the first and second CR, respectively. Melatonin phase was defined as the dim light melatonin onset calculated from $25 \%$ of the fitted three-harmonic peak-totrough amplitude (DLMO25\%) of the melatonin rhythm during the first CR. Melatonin suppression (mean $\pm \mathrm{SD}$ ) was calculated from the difference in the area under the curve (AUC), calculated using the trapezoidal method, between the melatonin profiles during the light exposure compared to the corresponding clock times during the previous melatonin cycle on CR1. Significance was assessed using one-tailed Student's t-tests. Absolute EEG power density values were expressed for each subject and light condition as a percentage of power density during dim light $(<2$ lux ) during an interval of equal clock time in the $\mathrm{CR}$ on the day prior to the light exposure. Log-transformed power densities were compared between the 460-nm and 555-nm monochromatic- light exposures with unpaired t-tests. Raw KSS ratings and performance parameters (mean and median reaction time, lapses [responses $>500 \mathrm{~ms}$ ]) measured during monochromatic-light exposure were subjected to two-way analysis of variance with time and wavelength as factors.

\section{Results}

Circadian phase-resetting

Monochromatic light exposure caused a phase delay of the melatonin rhythm in all subjects. Exposure to $6.5 \mathrm{~h}$ of $460 \mathrm{~nm}$ monochromatic light caused a significantly greater phase delay shift $(-2.98 \pm 0.50$ h) than did exposure to $555 \mathrm{~nm}$ monochromatic light $(-1.67 \pm 0.73 \mathrm{~h})(\mathrm{p}<0.001)$. When adjusted for the anticipated drift in phase due to circadian period [ $-0.4 \mathrm{~h}$ if $\tau=24.2 \mathrm{~h}$ (Czeisler et al., 1999)], $460 \mathrm{~nm}$ light caused twice as large a phase-shift as $555 \mathrm{~nm}$ light $(-2.58$ vs. $-1.27 \mathrm{~h}$ delay) despite equal photon densities.

\section{Melatonin suppression}

All subjects exposed to $460 \mathrm{~nm}$ monochromatic light had at least a $65 \%$ suppression of the melatonin AUC during the $6.5 \mathrm{~h}$ light exposure (range 65-96\%). Suppression was more variable among subjects exposed to $555 \mathrm{~nm}$ monochromatic light (0-88\%), including two individuals with no suppression. On average, exposure to $6.5 \mathrm{~h}$ of $460 \mathrm{~nm}$ monochromaticlight caused a significantly greater suppression of melatonin $(87.7 \pm 11.0 \% ; n=7)$ compared with 555 $\mathrm{nm}$ monochromatic light $(39.1 \pm 34.1 \% ; n=8)(p<$ 0.01) (Fig. 1). The time course of the response also differed between the two groups. Monochromatic light of $460 \mathrm{~nm}$ was able to suppress melatonin throughout the whole light exposure in all but one subject, who returned to the DLMO25\% level after $3.21 \mathrm{~h}$ of exposure (Fig. 1). Conversely, all but one subject exposed to $555 \mathrm{~nm}$ monochromatic light either failed to suppress to their DLMO25\% level at all $(n=3)$ or recovered to DLMO25\% after $\sim 2.6 \mathrm{~h}$

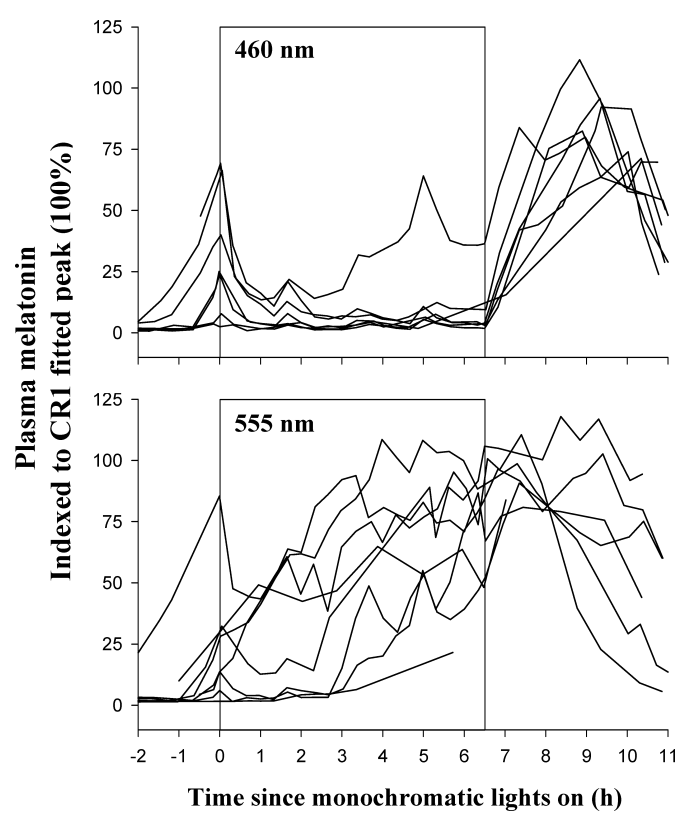

Fig. 1. Individual melatonin profiles before, during and after exposure to $460 \mathrm{~nm}$ or $555 \mathrm{~nm}$ monochromatic light, normalized to each individuals' fitted peak value during the first CR. Reproduced with permission from Lockley et al. (2003) J. Clin. Enocrinol. Metab. 

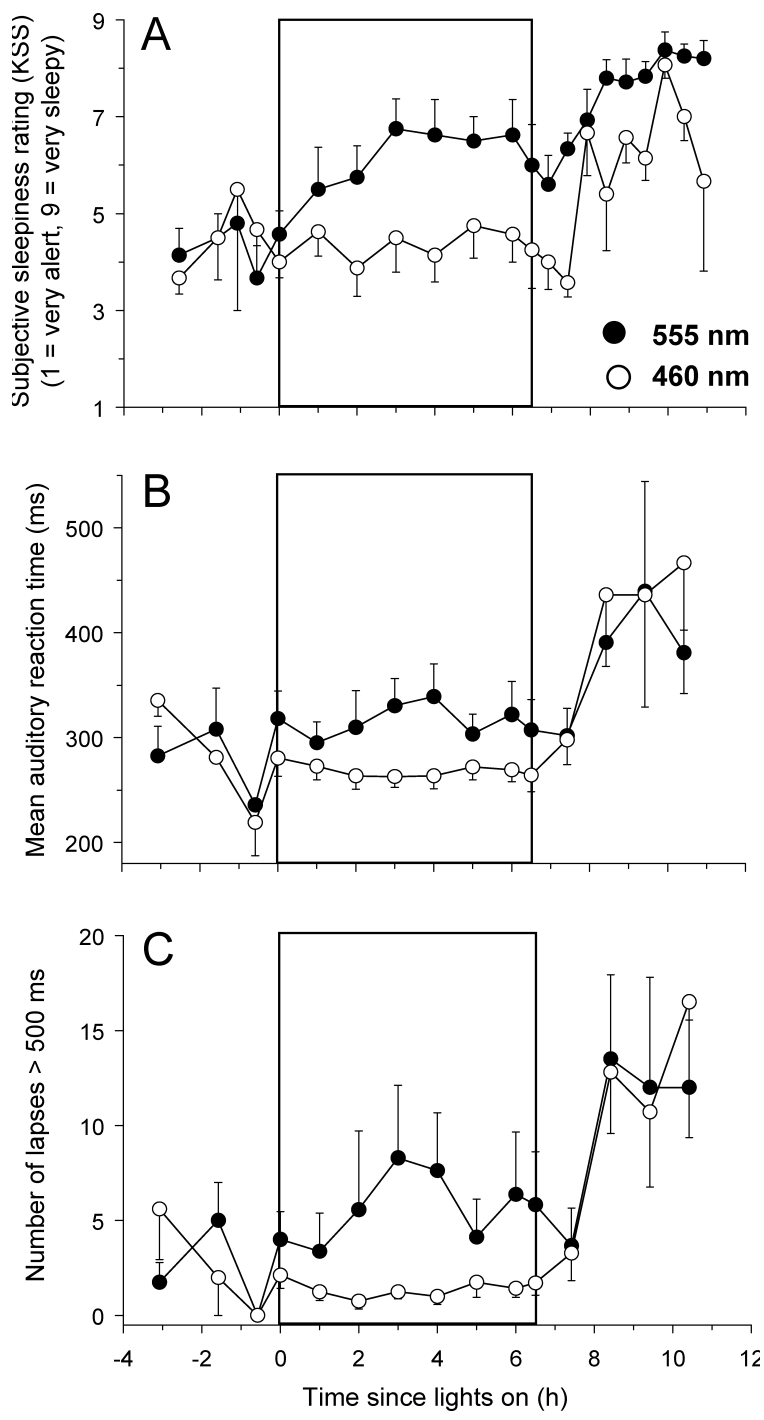

Fig. 2. Mean $( \pm \mathrm{SEM})$ group sleepiness, auditory reaction times and auditory lapses before, during and after exposure to monochromatic light for 6.5 hours $(\square)$. Exposure to $460-\mathrm{nm}$ light preferentially improved subjective sleepiness ratings, reaction time, and lapses of attention. Reproduced with permission from Lockley et al. (2006) Sleep.

$(n=4 ; 0.46,2.51,3.16,4.19 \mathrm{~h})$

\section{Subjective sleepiness}

There was no significant difference in KSS ratings between the two groups ( $460 \mathrm{~nm}, n=8 ; 555 \mathrm{~nm}, n=7$ ) when assessed at the onset of light exposure (Time 0 ) ( $p>0.05$, unpaired t-test) but during the light exposure, subjective sleepiness ratings during $460 \mathrm{~nm}$ exposure were significantly lower than during $555 \mathrm{~nm}$ exposure $(p<0.0001)$ (Fig. 2A). There was no effect of time or between time and wavelength.

\section{Auditory performance}

There was no significant difference in the performance variables measured between the two groups (460 nm, $n=8 ; 555 \mathrm{~nm}, n=7$ ) during the first light-

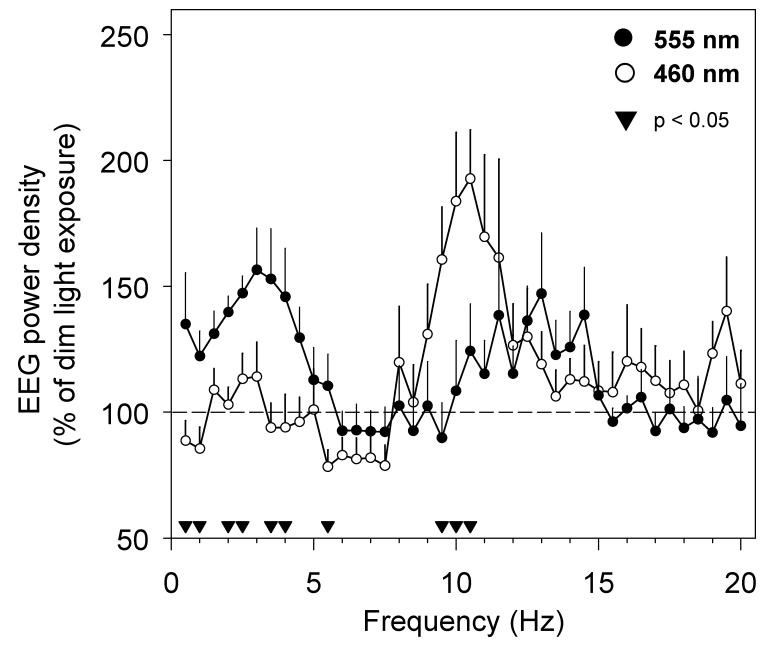

Fig. 3. Mean $( \pm$ SEM) group EEG power density during exposure to monochromatic light for 6.5 hours. Exposure to $460-\mathrm{nm}$ light $(\bigcirc)$ preferentially suppressed delta/theta activity and elevated high-alpha power as compared to $555 \mathrm{~nm}$ light (-). Significant differences for individual frequencies are shown by $\boldsymbol{\nabla}$. Reproduced with permission from Lockley et al., (2006) Sleep.

exposure test (Time 0) ( $p>0.05$, unpaired t-test). As shown in Fig. 2B, mean auditory reaction times were significantly faster during exposure to $460-\mathrm{nm}$ monochromatic light as compared to 555-nm light $(p<0.0001)$. There was no effect of time or between time and wavelength. A similar response was also observed for median auditory reaction time (data not shown). Auditory lapses in vigilance were also significantly reduced during exposure to $460 \mathrm{~nm}$ as compared with $555 \mathrm{~nm}$ light $(p<0.0001)$ (Fig. 2C).

\section{Waking EEG}

Exposure to light at $460 \mathrm{~nm}$ resulted in frequencyspecific changes in the waking EEG during Karolinksa Drowsiness Test (KDT) episodes, as compared with exposure to $555 \mathrm{~nm}$ (Fig. 3). Specifically, during exposure to $460 \mathrm{~nm}$, power densities in most bins in the frequency range of 0.5 to $5.5 \mathrm{~Hz}$ were reduced, and power densities in the 9.5- to 10.5$\mathrm{Hz}$ range were increased ( $p<0.05$, unpaired t-tests) (Fig. 3).

\section{Discussion}

Our study demonstrates that long-duration exposure to $460 \mathrm{~nm}$ light preferentially stimulates circadian phase resetting, suppression of melatonin, reduction of sleepiness, and improvements in auditory reaction time and lapses of attention as compared to exposure to an equal photon density of $555 \mathrm{~nm}$ light. This short-wavelength sensitivity indicates that the three-cone photopic visual system is not the primary photoreceptor system detecting light 
for these responses and is consistent with the hypothesis that these effects are mediated by a non-rod, noncone melanopsin-based photoreceptor system. These data are also consistent with the action spectra for short-duration melatonin suppression (Brainard et al., 2001; Thapan et al., 2001), and with the short-wavelength sensitivity demonstrated for circadian phase advance shifts (Warman et al., 2003), and the acute effects of light on subjective alertness ratings, performance, heart rate, and thermoregulation (Cajochen et al., 2005; Revell et al., 2006; Lehrl et al., 2007). While these studies are broadly consistent, there is some evidence to suggest, however, that not all of these effects of light have the same wavelength sensitivity and that these differences may depend on the time of day of exposure (Cajochen et al., 2005; Lockley et al., 2006; Revell et al., 2006). Additional work is required to elucidate fully the role of wavelength on the multiple effects of light, and how light timing, duration and intensity may affect the spectral sensitivity.

We also found that short wavelength light was able to change the power of electroencephalogram (EEG)derived frequencies to indicate a more alert state by preferentially suppressing delta/theta activity (0.5$5 \mathrm{~Hz}$ ) and enhancing high-alpha frequencies (9.5$10.5 \mathrm{~Hz}$ ) as compared to $555 \mathrm{~nm}$ exposure. Previous studies that have measured the acute effects of white light exposure on EEG power density have shown the major effect to be a suppression of activity in the theta-alpha range $(5-9 \mathrm{~Hz})$ (Cajochen et al., 2000). Changes in EEG power density in the delta/theta/ alpha frequency ranges $(0.5-9 \mathrm{~Hz})$ are influenced by both the circadian time of day and the duration of prior wakefulness (Aeschbach et al., 1997; 1999). High-frequency alpha activity $(9.25-12.0 \mathrm{~Hz})$, by contrast, does not exhibit a powerful wake-dependent component (Aeschbach et al., 1997) and has previously been shown to correlate negatively with the circadian changes in subjective sleepiness and circulating plasma melatonin levels (Aeschbach et al., 1999). The elevation of high alpha activity by short-wavelength light suggests that the effects of blue light might be mediated through inhibition of the circadian drive for sleep during the biological night. Whether this inhibition is achieved through suppression of melatonin and/or direct inhibition of the circadian drive for sleep emanating from the suprachiasmatic nuclei remains to be determined. Furthermore, the mechanism by which light improves alertness may not necessarily be the same as that which mediates melatonin suppression. The circadian rhythm of melatonin is closely correlated with subjective sleepiness (Aeschbach et al., 1999; Cajochen et al., 1999) and suppression of melatonin at night is usually accompanied by a simultaneous improvement in alertness (Cajochen et al., 2000). It is not known, however, whether light increases alertness by suppressing the sleep-promoting action of melatonin, or if alerting responses to light occur simultaneously and/or independently of melatonin suppression. Notably, light exposure during the day will improve alertness and performance when melatonin is undetectable (Phipps-Nelson et al., 2003; Rüger et al., 2006), suggesting that, at least during the biological day, alertness is not mediated via suppression of melatonin. Thus it may be possible that different nonvisual responses have differing spectral sensitivities to light, theoretically mediated through differing relative contributions of the short-wavelength sensitive melanopsin-driven system and the longer-wavelength sensitive photopic and scotopic visual photoreceptor systems (Lockley and Gooley, 2006).

Recent studies have begun to elucidate which neuronal pathways and brain structures mediate these 'non-visual' effects of light. Neuroanatomical investigations in rodents show that melanopsin-containing ganglion cells project to a range of retinorecipient nuclei including major projections to the hypothalamic suprachiasmatic nuclei (SCN), the site of endogenous circadian pacemaker; the intergeniculate leaflet of the thalamus, an area closely linked to normal circadian function which conveys photic and non-photic signals to the SCN; the ventral lateral preoptic area, an area which controls the switch between sleep and wake states; the olivary pretectal nucleus, implicated in the pupillary constriction response; and the superior colliculus, which mediates visual and auditory sensorimotor reponses (Gooley et al., 2003; Hattar et al., 2006). A subset of melanopsin-containing ganglion cells also project to the dorsal lateral geniculate nucleus (Dacey et al., 2005, Hattar et al., 2006), a key component of the visual system, suggesting that melanopsin-containing ganglion cells may also contribute to visual awareness. Recent imaging studies in humans are also beginning to identify brain regions associated with light-induced improvements in performance and cognition (Perrin et al., 2004; Vandewalle et al., 2006; Vandewalle et al., 2007) and show preferential short-wavelength activation of the thalamus and the anterior insula, structures implicated in arousal and memory function (Vandewalle et al., 2007).

Short-wavelength light has the potential to be developed for a range of therapeutic uses including treatment of sleep problems induced by circadian rhythm disorders (shift-work, jet-lag, advanced and delayed sleep phase syndrome, aging) or entrainment of the circadian system to unusual day-lengths (e.g., long-duration space flight, submariners, Antarctica). 
Preliminary studies have also shown that it can be used to treat Seasonal Affective Disorder (Glickman et al., 2006) and may be useful for correcting rest-activity disruption in dementia (Figueiro and Rea, 2005). The alerting effects of short-wavelength light also suggest that it can be used as a non-pharmacological countermeasure for drowsiness across a range of occupational, medical, educational and military settings. Given the widespread use of caffeine in modern society, countermeasures for sleepiness appear to be highly desired. For example, health professionals, pilots, public safety personnel (e.g., police, firefighters etc.) and shift-workers who are required to work $24 / 7$ would benefit from improved methodology to hasten adaptation to night work or to improve alertness while on duty. In addition, applications may be possible in standard occupational office settings to improve general day-time alertness or in educational settings such as colleges and schools where enhanced alertness may assist learning and memory. Furthermore, a countermeasure to provide sleepy car and truck drivers with a short burst of alertness that will allow them to find a safe place to stop driving and take a break would also be desirable. Caution is required, however, as these basic findings are translated into real-world applications. Until we fully understand the spectral sensitivity of all the effects of light on human physiology, including day-night variations and inter-individual differences, we may develop light exposure regimes that, while stimulating the system of interest, may inadvertently stimulate undesirable effects of light or inhibit desirable effects (Lockley, 2007). The challenge to architects and lighting designers is to incorporate these basic findings into lighting that optimizes both the visual and non-visual effects of light simultaneously and safely.

\section{Acknowledgements}

This work was supported by the National Institute of Neurological Disorders and Stroke (R01-NS36590 Brainard), the National Institute of Mental Health (R01-MH45130 Czeisler), the National Center for Complimentary and Alternative Medicine (R01AT002129 Lockley) and NASA Co-operative Agreement NCC9-58 with the National Space Biomedical Research Institute (Lockley, Brainard and Czeisler). The study was performed in the General Clinical Research Center, BWH which receives support from the National Center for Research Resources (M01RR02635).

A part of this study was presented at the 2nd International Symposium on Design of Artificial Environments which was held at Fukuoka in 2007.

\section{References}

Aeschbach, D., Matthews, J. R., Postolache, T. T., Jackson, M. A., Giesen, H. A., Wehr, T. A. (1997) Dynamics of the human EEG during prolonged wakefulness: evidence for frequency-specific circadian and homeostatic influences. Neurosci. Lett. 239: 121-124.

Aeschbach, D., Matthews, J. R., Postolache, T. T., Jackson, M. A., Giesen, H. A., Wehr, T. A. (1999) Two circadian rhythms in the human electroencephalogram during wakefulness. Am. J. Physiol. 277: R1771-1779.

Berson, D. M., Dunn, F. A., Takao, M. (2002) Phototransduction by retinal ganglion cells that set the circadian clock. Science 295: 1070-1073.

Brainard, G. C., Hanifin, J. P. (2005) Photons, clocks, and consciousness. J. Biol. Rhythms 20: 314-325.

Brainard, G. C., Hanifin, J. P., Greeson, J. M., Byrne, B., Glickman, G., Gerner, E., Rollag, M. D. (2001) Action spectrum for melatonin regulation in humans: Evidence for a novel circadian photoreceptor. J. Neurosci. 21: 6405-6412.

Cajochen, C., Jud, C., Munch, M., Kobialka, S., Wirz-Justice, A., Albrecht, U. (2006) Evening exposure to blue light stimulates the expression of the clock gene per2 in humans. Eur. J. Neurosci. 23: 1082-1086.

Cajochen, C, Khalsa, S. B., Wyatt, J. K., Czeisler, C. A., Dijk, D. J. (1999) EEG and ocular correlates of circadian melatonin phase and human performance decrements during sleep loss. Am. J. Physiol. 277: R640-649.

Cajochen, C., Munch, M., Kobialka, S., Krauchi, K., Steiner, R., Oelhafen, P., Orgul, S., Wirz-Justice, A. (2005) High sensitivity of human melatonin, alertness, thermoregulation, and heart rate to short wavelength light. J. Clin. Endocrinol. Metab. 90: 1311-1316.

Cajochen C., Zeitzer, J. M., Czeisler, C. A., Dijk, D. J. (2000) Dose-response relationship for light intensity and ocular and electroencephalographic correlates of human alertness. Behav. Brain Res. 115: 75-83.

Campbell, S. S., Dijk, D. J., Boulos, Z., Eastman, C. I., Lewy, A. J., Terman, M. (1995) Light treatment for sleep disorders: consensus report. III. Alerting and activating effects. J. Biol. Rhythms 10: 129-132.

Czeisler, C. A., Duffy, J. F., Shanahan, T. L., Brown, E. N., Mitchell, J. F., Rimmer, D. W., Ronda, J. M., Silva, E. J., Allan, J. S., Emens, J. S., Dijk, D. J., Kronauer, R. E. (1999) Stability, precision, and near-24-hour period of the human circadian pacemaker. Science 284: 21772181.

Czeisler, C. A., Shanahan, T. L., Klerman, E. B., Martens, H., Brotman, D. J., Emens, J. S., Klein, T., Rizzo, J. F., III (1995) Suppression of melatonin secretion in some blind patients by exposure to bright light. N. Engl. J. Med. 332: 6-11.

Czeisler, C. A., Wright Jr, K. P. (1999) Influence of light on circadian rhythmicity. In: Neurobiology of sleep and circadian rhythms, ed. by Turek, F. W. and Zee, P. C. Marcel Dekker Inc., New York, pp. 147-180.

Dacey, D. M., Liao, H. W., Peterson, B. B., Robinson, F. R., Smith, V. C., Pokorny, J., Yau, K. W., Gamlin, P. D. (2005) Melanopsin-expressing ganglion cells in primate retina signal colour and irradiance and project to the 
LGN. Nature 433: 749-754.

Duffy, J. F., Dijk, D. J. (2005) Getting through to circadian oscillators: why use constant routines? J. Biol. Rhythms 17: 4-13.

Figueiro, M. G., Rea, M. S. (2005) New research in the light and health field is expanding the possibilities for LED lighting in healthcare environments. In: CIE Midterm Meeting Conference Proceedings, Leon, Spain.

Glickman, G., Byrne, B., Pineda, C., Hauck, W. W., Brainard, G. C. (2006) Light therapy for seasonal affective disorder with blue narrow-band light-emitting diodes (LEDs). Biol. Psychiatry 59: 502-507.

Gooley, J. J., Lu, J., Chou, T. C., Scammell, T. E., Saper, C. B. (2001) Melanopsin in cells of origin of the retinohypothalamic tract. Nat. Neurosci. 4: 1165.

Gooley, J. J., Lu. J., Fischer, D., Saper, C. B. (2003) A broad role for melanopsin in nonvisual photoreception. J. Neurosci. 23: 7093-7106.

Hattar, S., Kumar, M., Park, A., Tong, P., Tung, J., Yau, K. W., Berson, D. M. (2006) Central projections of melanopsin-expressing retinal ganglion cells in the mouse. J. Comp. Neurol. 497: 326-349.

Hattar, S., Lucas, R. J., Mrosovsky, N., Thompson, S., Douglas, R. H., Hankins, M. W., Lem, J., Biel, M., Hofmann, F., Foster, R. G., Yau, K. W. (2003) Melanopsin and rodcone photoreceptive systems account for all major accessory visual functions in mice. Nature 424: 76-81.

Hull, J. T., Lockley, S. W., Dijk, D. J., Czeisler, C. A. (2004) Non-24-hour sleep-wake disorder in a totally blind individual with intact circadian photoreception. J. Sleep Res. 13(S1): 347.

Klerman, E. B., Shanahan, T. L., Brotman, D. J., Rimmer, D. W., Emens, J. S., Rizzo III, J. F., Czeisler, C. A. (2002) Photic resetting of the human circadian pacemaker in the absence of conscious vision. J. Biol. Rhythms 17: 548-555.

Lehrl, S., Gerstmeyer, K., Jacob, J. H., Frieling, H., Henkel, A. W., Meyrer, R., Wiltfang, J., Kornhuber, J., Bleich, S. (2007) Blue light improves cognitive performance. J. Neural Transmission 114: 457-460.

Lockley, S. W. (2007) Safety considerations for the use of blue-light blocking glasses in shift-workers. J. Pineal Res. 42: 210-1.

Lockley, S. W., Brainard, G. C., Czeisler, C. A. (2003) High sensitivity of the human circadian melatonin rhythm to resetting by short wavelength light. J. Clin. Endocrinol. Metab. 88: 4502-4505.

Lockley, S. W., Evans, E. E., Scheer, F. A., Brainard, G. C., Czeisler, C. A., Aeschbach, D. (2006) Short-wavelength sensitivity for the direct effects of light on alertness, vigilance and waking electroencephalogram in humans. Sleep 29: 161-168.

Lockley, S. W., Gooley, J. J. (2006) Circadian photoreception: Spotlight on the brain. Curr. Biol. 16: R795-797.

Lockley, S. W., Skene, D. J., Arendt, J., Tabandeh, H., Bird,
A. C., Defrance, R. (1997) Relationship between melatonin rhythms and visual loss in the blind. J. Clin. Endocrinol. Metab. 82: 3763-3770.

Phipps-Nelson, J., Redman, J. R., Dijk, D. J., Rajaratnam, S. M. (2003) Daytime exposure to bright light, as compared to dim light, decreases sleepiness and improves psychomotor vigilance performance. Sleep 26: 695 700.

Peirson, S., Foster, R. G. (2006) Melanopsin: another way of signaling light. Neuron 49: 331-339.

Perrin, F., Peigneux, P., Fuchs, S., Verhaeghe, S., Laureys, S., Middleton, B., Degueldre, C., Del Fiore, G., Vandewalle, G., Balteau, E., Poirrier, R., Moreau, V., Luxen, A., Maquet, P., Dijk, D. J. (2004) Nonvisual responses to light exposure in the human brain during the circadian night. Curr. Biol. 14: 1842-1846.

Provencio, I., Rodriguez, I. R., Jiang, G., Hayes,W. P., Moreira, E. F., Rollag, M. D. (2000) A novel human opsin in the inner retina. J. Neurosci. 20: 600-605.

Revell, V. L., Arendt, J., Fogg, L. F., Skene, D. J. (2006) Alerting effects of light are sensitive to very short wavelengths. Neurosci. Lett. 399: 96-100.

Ruberg, F. L., Skene, D. J., Hanifin, J. P., Rollag, M. D., English, J., Arendt, J., Brainard, G. C. (1996) Melatonin regulation in humans with color vision deficiencies. J. Clin. Endocrinol. Metab. 81: 2980-2985.

Rüger, M., Gordijn, M. C., Beersma, D. G., De Vries, B., Daan, S. (2006) Time-of-day-dependent effects of bright light exposure on human psychophysiology: comparison of daytime and nighttime exposure. Am. J. Physiol. 290: R1413-1420.

Thapan, K., Arendt, J., Skene, D. J. (2001) An action spectrum for melatonin suppression: evidence for a novel non-rod, non-cone photoreceptor system in humans. J. Physiol. 535: 261-267.

Vandewalle, G., Balteau, E., Phillips, C., Degueldre, C., Moreau, V., Sterpenich, V., Albouy, G., Darsaud, A., Desseilles, M., Dang-Vu, T. T., Peigneux, P., Luxen, A., Dijk, D. J., Maquet, P. (2006) Daytime light exposure dynamically enhances brain responses. Curr. Biol. 16: $1616-1621$

Vandewalle, G., Gais, S., Schabus, M., Balteau, E., Carrier, J., Darsaud, A., Sterpenich, V., Albouy, G., Dijk, D., Maquet, P. (2007) Wavelength-dependent modulation of brain responses to a working memory task by daytime light exposure. Cereb. Cortex. Advance Access published online on April 2, 2007, doi:10.1093/cercor/ bhm007.

Warman, V. L., Dijk, D. J., Warman, G. R., Arendt, J., Skene, D. J. (2003) Phase advancing human circadian rhythms with short wavelength light. Neurosci. Lett. 342: 37-40.

Zeitzer, J. M., Dijk, D. J., Kronauer, R., Brown, E., Czeisler, C. (2000) Sensitivity of the human circadian pacemaker to nocturnal light: melatonin phase resetting and suppression. J. Physiol. 526: 695-702. 